\title{
A Three-Pole Substrate Integrated Waveguide Bandpass Filter Using New Coupling Scheme
}

\author{
Xuehui GUAN, Ye YUAN, Haiwen LIU, Wei HUANG \\ School of Information Engineering, East China Jiaotong University, 330013 Nanchang, P.R. China
}

guan@ecjtu.edu.cn

\begin{abstract}
A novel three-pole substrate integrated waveguide (SIW) bandpass filter (BPF) using new coupling scheme is proposed in this paper. Two high order degenerate modes ( $T E_{102}$ and $\left.T E_{201}\right)$ of a square SIW cavity and a dominant mode (TE ${ }_{101}$ ) of a rectangular SIW cavity are coupled to form a three-pole SIW BPF. The coupling scheme of the structure is given and analyzed. Due to the coupling between two cavities, as well as the coupling between source and load, three transmission zeros are created in the stopband of the filter. The proposed threepole SIW BPF is designed and fabricated. Good agreement between simulated and measured results verifies the validity of the design methodology well.
\end{abstract}

\section{Keywords}

Transmission zero, substrate integrated waveguide (SIW), degenerate mode, coupling matrix

\section{Introduction}

Substrate integrated waveguide (SIW) has gained wide attention for its advantages of low cost, high Q, and easy connection with planar devices. SIW components, such as filters, couplers, diplexers and so on, have been widely used in various microwave and millimeter wave systems [1], [2]. Traditionally, SIW filters can be implemented by inserting irises in a SIW. Desired inductor and capacitor effect can be realized by controlling the irises [3]. In [4], a four-pole bandpass filter (BPF) is realized by using four side-by-side horizontally oriented SIW cavities. Each cavity acts as a resonator and they are coupled to form a bandpass characteristic. Cavities also can be arranged in an extended box configuration with dispersive coupling on a main signal path, which produces an extra zero in comparison to classical approaches [5]. Dominant resonant mode ( $\mathrm{TE}_{101}$ mode) of the SIW cavities is applied in these SIW filters. Degenerated modes of SIW cavity can also be used in the filter. In [6], a dual-mode SIW BPF has been proposed by using orthogonal feed lines and two slot lines for disturbing two degenerate modes. To realize a sharp transition band and improve selectivity of the filter, high order or multi-pole filters are needed. In [7], a planar SIW elliptic BPF is investigated by cascading two extend doublets. Multi-pole BPFs are realized by etching a multimode microstrip resonator on the top metal layer or on the bottom ground layer of a SIW resonator in [8] and [9]. Although it can achieve multi-pole bandpass characteristic, slot on the metal layer of the SIW cavity may cause radiation and worsen characteristic of the filter. In [10], an inline and a folded three-pole dual-mode SIW filters with asymmetric transmission response are presented. Unlike the SIW filters with resonant cavities [11], the signal is coupled to different modes by eccentrically fed SIW, which provides a new way to realize multi-pole SIW BPF.

In this paper, a novel three-pole SIW BPF is proposed by using two SIW cavities with dissimilar sizes. Two degenerate modes $\left(\mathrm{TE}_{102}\right.$ and $\left.\mathrm{TE}_{201}\right)$ of the bigger square SIW cavity and a dominant mode $\left(\mathrm{TE}_{101}\right)$ of the smaller rectangular SIW cavity are coupled together to form a three-pole SIW BPF. By virtue of source-load coupling and the coupling between two SIW cavities, three transmission zeros are realized, which improve the performance of the proposed SIW BPF. Finally, the SIW BPF is designed, fabricated and measured. Good agreement between simulation and measurement verifies the validity of the design methodology.

\section{Analysis and Design}

\subsection{Analysis of the SIW Filter}

Figure 1 shows the configuration of the proposed three-pole SIW BPF. It mainly consists of a square SIW cavity (Cavity 1) and a rectangular SIW cavity (Cavity 2). The filter is fed by a pair of microstrip lines via the coplanar waveguide (CPW) transition structure.

The size of SIW cavity is determined by the corresponding resonant frequency. The resonant frequency of the resonant modes $\left(\mathrm{TE}_{m 0 n}\right)$ of the SIW dual-mode filter can be calculated using the following equation [12]:

$$
f_{r}=\frac{c_{0}}{2 \sqrt{\varepsilon_{r}}}=\sqrt{\left(\frac{m}{W_{e f f}}\right)^{2}+\left(\frac{n}{L_{e f f}}\right)^{2}}
$$

where

$$
W_{\text {eff }}=L_{\text {eff }}=W-\frac{D^{2}}{0.95 \cdot S}=L-\frac{D^{2}}{0.95 \cdot S}
$$


where $m$ and $n$ are the indices of the modes, $\varepsilon_{\mathrm{r}}$ is the permittivity of the substrate material, and $c_{0}$ is the velocity of the light in free space, $D$ is the diameter of the metal via, $W$ and $L$ are the width and length of the cavity, respectively, $S$ is the space between metal vias, $W_{\text {eff }}$ and $L_{\text {eff }}$ are the equivalent width and length of the rectangular cavity, respectively.

As to a square SIW cavity, its $\mathrm{TE}_{201}$ mode and $\mathrm{TE}_{102}$ mode has the same resonant frequency. To ensure that the dominant mode of cavity 2 has the same resonant frequency with the high order mode of cavity 1, according to (1), side length of cavity 2 is set about half of longitudinal dimension.

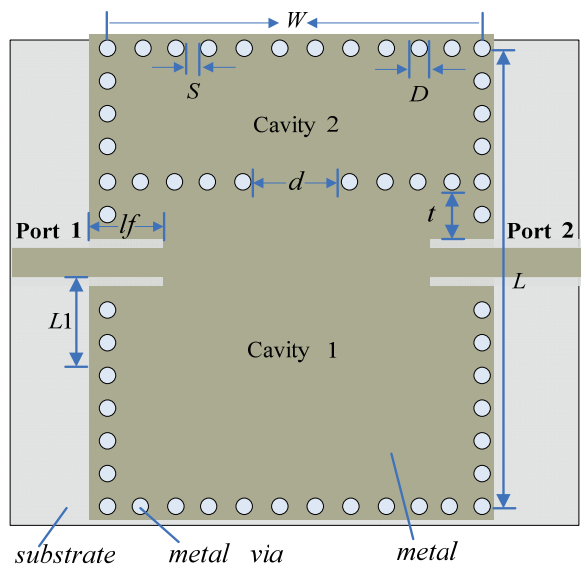

Fig. 1. Layout of proposed three-pole SIW BPF.

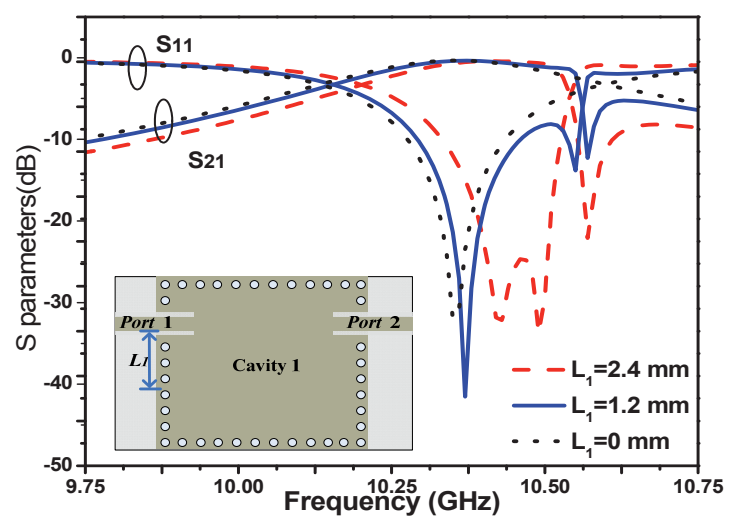

Fig. 2. Simulated characteristics of cavity 1 against $L_{1}$.

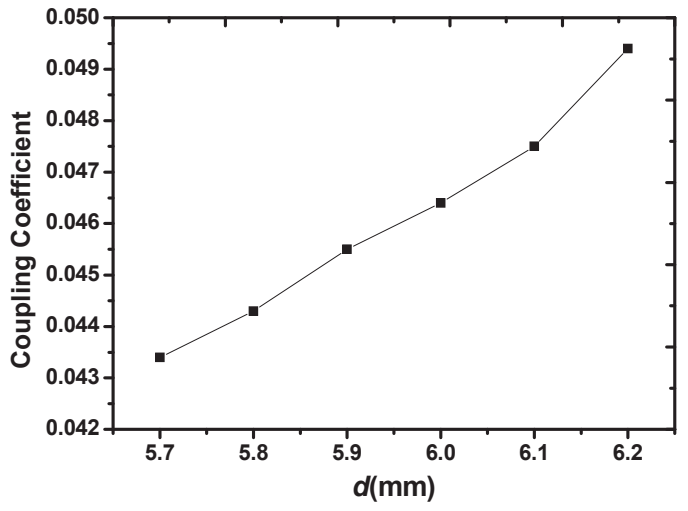

Fig. 3. The coupling between cavity 1 and cavity 2 versus $d$.
In order to excite the degenerate modes of cavity 1 , feed lines of the dual-mode SIW resonator offset from the center of cavity 1 [13]. Figure 2 illustrates the bandpass characteristics of the dual-mode SIW cavity against offset length $L_{1}$. When $L_{1}=0 \mathrm{~mm}$, two degenerate modes of cavity 1 coincide with each other and only one resonant peak can be observed. When $L_{1}$ increases from 0 to $2.4 \mathrm{~mm}$, its two degenerate modes are split and the other resonant peak can also be seen. The coupling strength between cavity 1 and cavity 2 is controlled by the width of iris $d$. Figure 3 gives the simulated coupling coefficient between cavity 1 and cavity 2 versus $d$. When $d$ increases from 5.7 to $6.2 \mathrm{~mm}$, the coupling coefficients increases from 0.0435 to 0.0495. In addition, because two degenerate modes of cavity 1 are coupled with each other, the coupling scheme of the proposed filter can be affirmed.

Figure 4 shows the coupling scheme of the proposed three-pole SIW BPF. Two degenerated modes of cavity 1 are directly coupled to both source and load, and the dominant resonant mode of cavity 2 is coupled to one degenerated mode of cavity 1 . Corresponding coupling matrix of coupling scheme of the proposed filter can be written as

$$
M=\left[\begin{array}{ccccc}
0 & M_{s 1} & M_{s 2} & 0 & M_{S L} \\
M_{s 1} & 0 & M_{12} & 0 & M_{1 L} \\
M_{s 2} & M_{12} & 0 & M_{23} & M_{2 L} \\
0 & 0 & M_{23} & 0 & 0 \\
M_{S L} & M_{1 L} & M_{2 L} & 0 & 0
\end{array}\right]
$$

where $M_{\mathrm{S} 1}$ and $M_{\mathrm{S} 2}$ represent the coupling between source and degenerate modes of cavity $1, M_{1 \mathrm{~L}}$ and $M_{2 \mathrm{~L}}$ indicate the coupling between load and degenerate modes of cavity 1 , $M_{12}$ indicates the mutual coupling between the two degenerate modes, and $M_{23}$ denotes the coupling between the main mode of the cavity 2 and one degenerate mode of cavity 1 . Coupling matrix of the filter is synthesized as [14]:

$$
M=\left[\begin{array}{ccccc}
0 & 0.5249 & 0.4545 & 0 & -0.021 \\
0.5249 & 0 & -0.09 & 0 & -0.61 \\
0.4545 & -0.09 & 0 & -0.76 & 0.5249 \\
0 & 0 & -0.76 & 0 & 0 \\
-0.021 & -0.61 & 0.5249 & 0 & 0
\end{array}\right]
$$

Figure 5 presents the synthesized scattering characteristics of the proposed filter. The in-band return loss is about $16 \mathrm{~dB}$. Two transmission zeros are achieved in the transition band, improving the selectivity of the filter. Outof-band rejection at both sides is higher than $15 \mathrm{~dB}$.

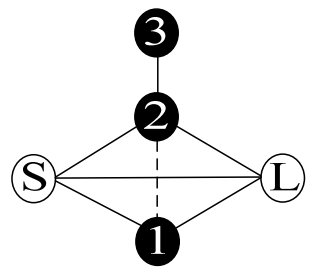

Fig. 4. Coupling scheme of the proposed three-pole SIW BPF. 


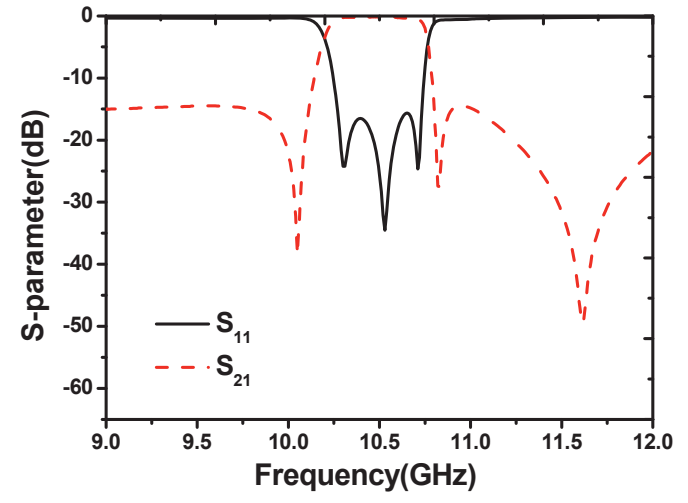

Fig. 5. The synthesized scattering characteristics of the proposed filter.

\subsection{Discussion on Characteristics of the Filter}

Figure 6 gives a comparison of transmission characteristics between dual-mode SIW filter, original three-pole filter, and the proposed filter with source-load (SL) coupling. Three transmission zeros are clearly observed in the stopband of the proposed filter with SL coupling. Comparing the transmission characteristics of these structures, a conclusion can be drawn that a transmission zero, namely $\mathrm{TZ}_{2}$, will be created in the higher stopband of the filter by virtue of dual-mode SIW cavity [14]. Extended coupled cavity 2 produce a transmission zero $\left(\mathrm{TZ}_{1}\right)$ in the lower stopband of the filter. And the third transmission zero $\mathrm{TZ}_{3}$ is mainly attributed to the coupling between source and load. To verify the above-mentioned analysis, the relation between transmission zeros and parameters of the filter are studied.

Originally, when two degenerated modes of cavity 1 are split, it provides two signal paths from source to load. It should be noted that when the signal from two paths are of same magnitude but opposite phase, transmission zero $\mathrm{TZ}_{2}$ will be created. When cavity 2 is attached and coupled to cavity 1 , additional transmission zero $\mathrm{TZ}_{1}$ is achieved. Figure 7(a) depicts the transmission characteristics of the filter against iris width $d$. When iris width $d$ increases from $5.4 \mathrm{~mm}$ to $6 \mathrm{~mm}, \mathrm{TZ}_{1}$ shifts down from $10.16 \mathrm{GHz}$ to $9.97 \mathrm{GHz}$, while $\mathrm{TZ}_{2}$ and $\mathrm{TZ}_{3}$ almost keep unchanged. Obviously, $\mathrm{TZ}_{1}$ can be controlled by changing iris width $d$. Meanwhile, it is clearly observed that the low edge of the passband moves down and the bandwidth of the filter increases with the increment of $d$.

Figure 7(b) presents the transmission characteristics of the filter against $l_{\mathrm{f}}$, the probe depth of the CPW. When $l_{\mathrm{f}}$ increases from $4 \mathrm{~mm}$ to $6 \mathrm{~mm}$, transmission zero $\mathrm{TZ}_{3}$ moves from $11.9 \mathrm{GHz}$ to $11.4 \mathrm{GHz}$, and $\mathrm{TZ}_{1}$ decreases from $10.1 \mathrm{GHz}$ to $10 \mathrm{GHz}$, while $\mathrm{TZ}_{2}$ keeps almost unchanged. The $\mathrm{CPW}$ transition structure provides a coupling between source and load, which increases with increment of $l_{\mathrm{f}}$. Furthermore, the increment of $l_{\mathrm{f}}$ will change the coupling between degenerated modes and source/load, which leads to the shift of $\mathrm{TZ}_{1}$, but has a slight effect on the bandwidth of the filter.

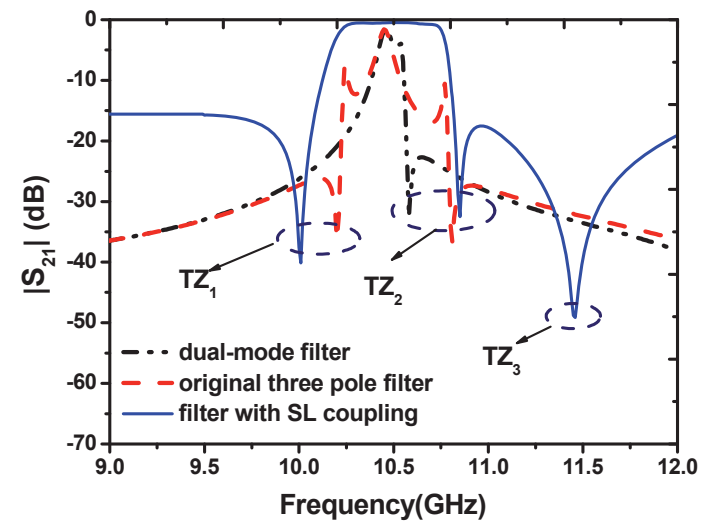

Fig. 6. A comparison of transmission characteristics between dual-mode SIW filter, original three-pole filter, and the proposed filter with source-load (SL) coupling.

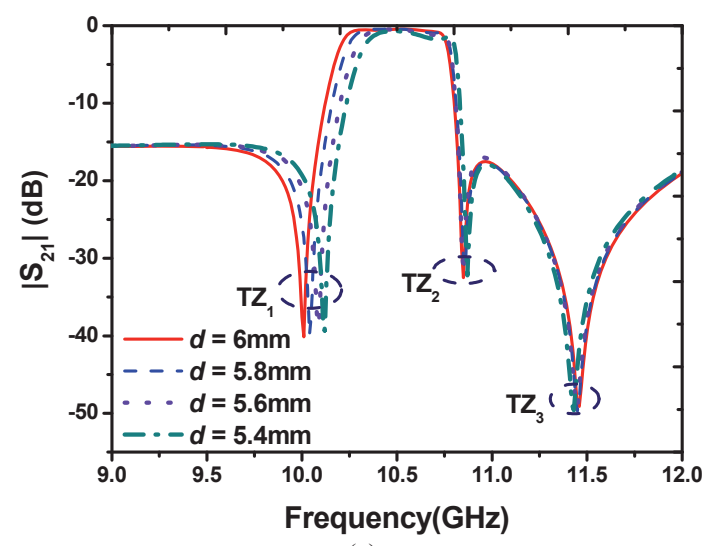

(a)

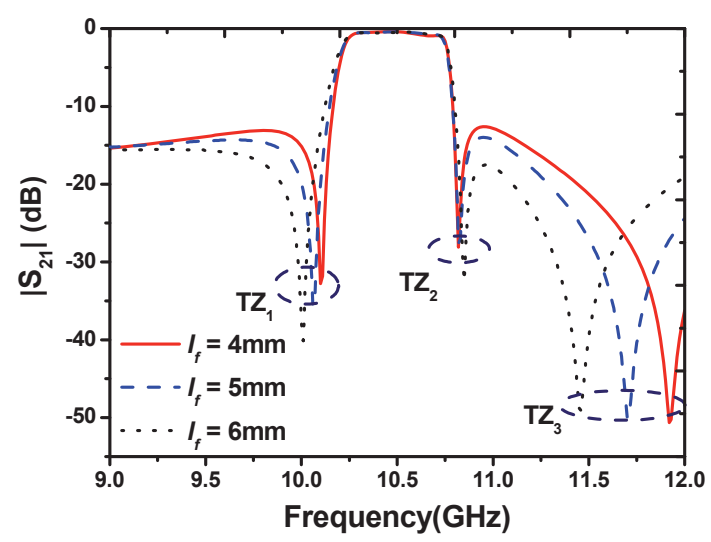

(b)

Fig. 7. Simulated transmission characteristics of the filter against (a) $d$, and (b) $l_{\mathrm{f}}$

Figure 8 presents the reflection characteristics of the filter against $t$. When $t$ increases from $5.6 \mathrm{~mm}$ to $6 \mathrm{~mm}$, transmission poles move away from each other and the bandwidth of the filter enlarged accordingly.

Obviously, from the coupling structure and the coupling matrix of the filter, the larger coupling between resonant modes will cause larger bandwidth [14]. Parametric study also shows that the bandwidth of the filter can be tuned by modifying $d$ and $l_{\mathrm{f}}[15]$. 


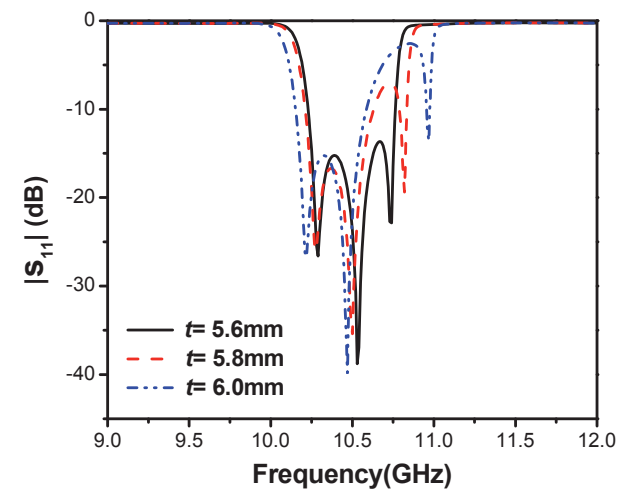

Fig. 8. Simulated reflection characteristics of the filter against $t$.

\section{Experimental Results}

Based on the proposed structure and coupling scheme, a novel three-pole SIW BPF with central frequency of $10.5 \mathrm{GHz}$ is designed and fabricated. The designed filter has an equal ripple of $0.15 \mathrm{~dB}$ in the passband and fractional bandwidth is $4.8 \%$. The filter is fabricated on a Rogers RT/duroid 5880 substrate with a relative dielectric constant of 2.2, thickness of $0.508 \mathrm{~mm}$, and loss tangent of 0.0009 . Finally, the obtained parameters of the filter shown in Fig. 1 are: $D=0.6 \mathrm{~mm}, S=0.3 \mathrm{~mm}$, $W=22 \mathrm{~mm}, \quad L=32.65 \mathrm{~mm}, \quad L_{1}=2.2 \mathrm{~mm}, l_{\mathrm{f}}=5.8 \mathrm{~mm}$, $d=5.9 \mathrm{~mm}$, and $t=5.6 \mathrm{~mm}$.

The filtering performance is measured by using Network Analyzer AV3926. A comparison between EM simulated results and measured results are shown in Fig. 9. Solid lines and dashed lines indicate the simulated and

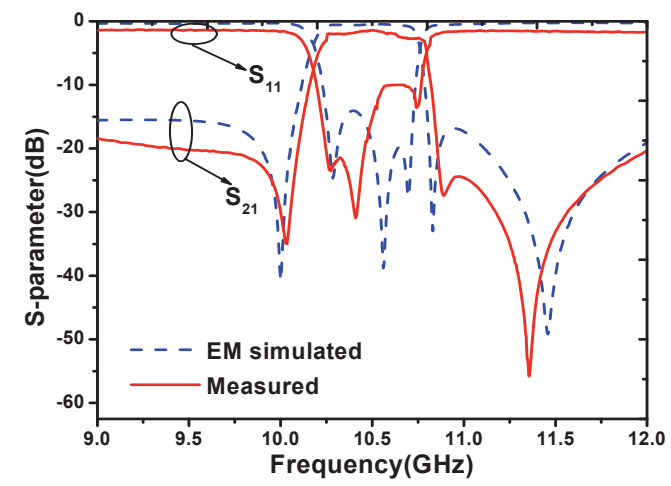

Fig. 9. A comparison between EM simulated results and measured results.

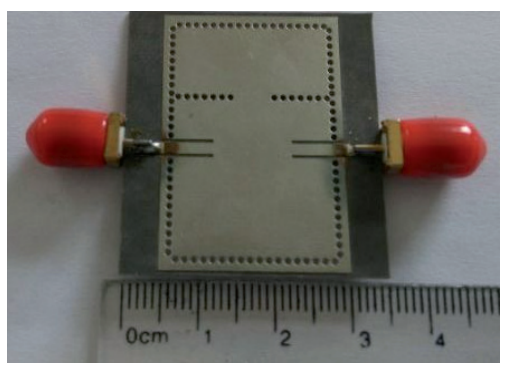

Fig. 10. A photograph of the fabricated filter. measured results, respectively. The passband of the proposed filter covers from $10.25 \mathrm{GHz}$ to $10.75 \mathrm{GHz}$, and its return loss is better than $15 \mathrm{~dB}$. Three transmission poles are clearly observed at $10.28 \mathrm{GHz}, 10.57 \mathrm{GHz}$, and $10.78 \mathrm{GHz}$. Three transmission zeros are created at $9.97 \mathrm{GHz}, 10.86 \mathrm{GHz}$, and $11.35 \mathrm{GHz}$, which improve the stopband characteristics of the filter. Simulated and measured insertion loss in the passband is $0.76 \mathrm{~dB}$ and $1.39 \mathrm{~dB}$, respectively. The relatively large insertion loss is mainly attributed to the SMA connections and the test transitions used in the measurement. Two $3.5 \mathrm{~mm}$-to- $2.4 \mathrm{~mm}$ transitions are additionally used while performing transition from $2.4 \mathrm{~mm}$ of Network Analyzer to $3.5 \mathrm{~mm}$ SMA. A photograph of the fabricated filter is shown in Fig. 10.

\section{Conclusion}

In this article, a novel three-pole SIW BPF with new coupling scheme is proposed. The filter is consisted by a square SIW cavity and a rectangular SIW cavity. Two degenerate modes of the square SIW cavity and a main mode of the rectangular SIW cavity are coupled together to form a BPF. Three transmission zeros are created in the stopband of the filter, which improve the stopband characteristics of the proposed filter.

\section{Acknowledgments}

This work is financially supported by the NSFC (No. 61161005, 61461020), 555 Talent Program of Jiangxi Province, Jiangxi Provincial Natural Science Foundation (No. 20152ACB21007), and Jiangxi Provincial Sailing Project, all in China.

\section{References}

[1] CHE, W. Q., LEI, X., WANG, D. P., et al. Equivalence between substrate-integrated (SIRW) rectangular waveguide short-circuit load and its equivalent rectangular waveguide short-circuit load. Microwave and Optical Technology Letters, 2006, vol. 48, no. 9, p. 1694-1698. DOI: 10.1002/mop.21805

[2] CHE, W. Q., DENG, K., YUNG, E. K. N., et al. H-plane 3-dB hybrid ring of high isolation in substrate integrated rectangular waveguide. Microwave and Optical Technology Letters, 2006, vol. 48, no. 3, p. 502-505. DOI: 10.1002/mop.21392

[3] CHEN, X. P., DROLET, D., WU, K. Substrate integrated waveguide filters for airborne and satellite system applications. In $\mathrm{Ca}$ nadian Conf. on Electrical and Computer Engineering. Vancouver (Canada), 2007, p. 659-662. DOI: 10.1109/CCECE.2007.169

[4] SHEN, W., YIN, W. Y., SUN, X. W., et al. Compact coplanar waveguide-incorporated substrate integrated waveguide filter. Journal of Electromagnetic Waves and Application, 2010, vol. 24, no. 7, p. 871-879. DOI: 10.1163/156939310791285164

[5] SZYDLOWSKI, L., LESZCZYNSKA, N., LAMECKI, A., et al. A substrate integrated waveguide (SIW) bandpass filter in a box configuration with frequency-dependent coupling. IEEE 
Microwave and Wireless Components Letters, 2012, vol. 22, no. 11, p. 556-558. DOI: 10.1109/LMWC.2012.2221690

[6] LI, R. Q., TANG, X. H., XIAO, F. Substrate integrated waveguide dual-mode filter using slot lines perturbation. Electronics Letters, 2010 , vol. 46 , no. 12 , p. $845-846$. DOI: 10.1049/el.2010.0629

[7] SHEN, K., WANG, G. M., FU, S. H., GU, G. D., Highly selective bandpass filter based on substrate integrated waveguide. Electronics Letters, 2009, vol. 45, no. 14, p. 746-748. DOI: 10.1049/el.2009.0785

[8] CHEN, X. P., HONG, W., CUI, T., CHEN, J., WU, K. Substrate integrated waveguide (SIW) linear phase filter. IEEE Microwave and Wireless Components Letters, 2005, vol. 15, no. 11, p. 787 to 789. DOI: 10.1109/LMWC.2005.859021

[9] SHEN, W., YIN, W. Y., SUN, X. W. Compact substrate integrated waveguide (SIW) filter with defected ground structure. IEEE Microwave and Wireless Components Letters, 2011, vol. 21, no. 2, p. 83-85. DOI: 10.1109/LMWC.2010.2091402

[10] CHEN, X. P., HAO, Z. C., HONG, W., et al. Planar asymmetric dual-mode filters based on substrate integrated waveguide (SIW). In IEEE MTT-S International Microwave Symposium Digest. USA 2005, p. 12-17. DOI: 10.1109/MWSYM.2005.1516782

[11] CHEN, X.-P., WU, K., LI, Z.-L. Dual-band and triple-band substrate integrated waveguide filters with Chebyshev and quasielliptic responses. IEEE Transactions on Microwave Theory and Technique, 2007, vol. 55, no. 12, p. 2569-2578. DOI: 10.1109/TMTT.2007.909603

[12] DESLANDES, D., WU, K. Substrate integrated waveguide dualmode filters for broadband wireless systems. In Proceedings of Radio and Wireless Conference RAWCON. Boston (USA), 2003, p. 385-388. DOI: 10.1109/RAWCON.2003.1227973

[13] ALMALKAWI, M., ZHU, L., DEVABHAKTUNI, V. Dual-mode substrate integrated waveguide (SIW) bandpass filters with an improved upper stopband performance. In Proceedings of 36th International Conference on Infrared, Millimeter and Terahertz Waves IRMMW-THz 2011. Houston (USA), 2011, p. 1-2. DOI: 10.1109/irmmw-THz.2011.6105128

[14] AMARI, S., ROSENBERG, U., BORNEMANN, J. Adaptive synthesis and design of resonators with source/load-multiresonator coupling. IEEE Transactions on Microwave Theory and Technique, 2002, vol. 50, no. 5, p. 1969-1978. DOI: 10.1109/TMTT.2002.801348

[15] SHEN, W., YIN, W. Y., SUN, X. W. Miniaturized dual-band substrate integrated waveguide filter with controllable bandwidths. IEEE Microwave and Wireless Components Letters, 2011, vol. 21, no. 8, p. 418-420. DOI: 10.1109/LMWC.2011.2158412

\section{About the Authors ...}

Xuehui GUAN was born in Jiangxi, P.R. China. He received his Ph.D degree from Shanghai University in 2007.
Since June 2007, he has been an Associate Professor with the School of Information Engineering, East China Jiaotong University, Nanchang, China. In 2012, he was a Senior Researcher Associate with the School of Electrical and Electronic Engineering, City University of Hong Kong, Kowloon, Hong Kong. Since June 2013, he has been a Visiting Scholar with the School of Electrical and Electronic Engineering, Nanyang Technological University, Singapore. His current research interests include radio frequency and microwave passive circuits and systems, synthesis theory and realization of microwave filters, and antennas for wireless communications.

Ye YUAN was born in Hunan. He received the B.S. degree from Hunan Institute of Science and Technology in 2011. Now he is working toward his Master Degree in East China Jiaotong University. His current research interests include multi-mode microwave filters.

Haiwen LIU received the B.S. degree in Electronic System and the M.S. degree in Radio Physics from Wuhan University, Wuhan, China, in 1997 and 2000, respectively, and the Ph.D. degree in Microwave Engineering from Shanghai Jiao Tong University, Shanghai, China, in 2004. From 2004 to 2006, he was with Waseda University, Kitakyushu, Japan, as a Research Assistant Professor. From 2006 to 2007, he was a Research Fellow with Kiel University, Kiel, Germany, where he was granted the Alexander von Humboldt Research Fellowship. From 2007 to 2008, he was a Professor with the Institute of Optics and Electronics, Chengdu, China, where he was supported by the 100 Talents Program of Chinese Academy of Sciences. Since 2009, he has been a Chair Professor with East China Jiaotong University, Nanchang, China. He has published more than 100 papers in international and domestic journals and conferences. His current research interests include electromagnetic modeling of high-temperature superconducting circuits, radio frequency and microwave passive circuits and systems, synthesis theory and practices of microwave filters and devices, antennas for wireless terminals, and radar system.

Wei HUANG was born in Jiangxi. He received the B.S. degree from Jiangxi University of Finance and Economics in 2014. Now he is working toward his Master Degree in East China Jiaotong University. His current research interests include microstrip filters and SIW. 